\title{
Analyse De Quelques Parametres De Consommation De Wavé-Fortex, Un Complement Alimentaire Naturel Du Gabon
}

\author{
Alexis Nicaise Lepengue \\ Laboratoire des Phyto-alicaments ; Département de Biologie ; Université des \\ Sciences et Techniques de Masuku (USTM). Franceville, Gabon \\ Jean Fabrice Yala \\ Laboratoire de Microbiologie ; Département de Biologie ; Université des \\ Sciences et Techniques de Masuku (USTM). Franceville, Gabon
}

\section{Alain Souza}

Laboratoire de Physiologie Animale-Pharmacologie ; Département de

Biologie ; Université des Sciences et Techniques de Masuku (USTM).

Franceville, Gabon

\section{Bertrand Mbatchi}

Laboratoire des Phyto-alicaments ; Département de Biologie ; Université des

Sciences et Techniques de Masuku (USTM). Franceville, Gabon

Doi: 10.19044/esj.2018.v14n24p193 URL:http://dx.doi.org/10.19044/esj.2018.v14n24p193

\begin{abstract}
Wavé-fortex is a food supplement developed by Masuku University of Science and Technology (USTM) in Gabon. It aims at fighting against metabolic diseases, especially impotence, fatigue and sexual weakness, hypertension, overweight and cardiac arrhythmias. This study was conducted in order to analyze the distribution of consumption by type of diseases. The results revealed that Wavé-fortex is mainly consumed for problems of sexual dysfunction $(41 \%)$, general fatigue $(20 \%)$ and hypertension $(15 \%)$. In terms of gender, men are the main consumers at $73 \%$. Their consumption is mainly due to sexual stability concerns (51\%), general fatigue (24\%) and hypertension (23\%). Among women, the concerns are rather overweight (32\%) and hypertension (29\%), before sexual disorders (21\%). Wavé-fortex is a product mainly consumed in Gabon (70\%), Congo (14\%), Cameroon (6\%) and France $(2.5 \%)$, by patients aged between 30 and 70 years.
\end{abstract}

Keywords: Wavé-fortex; foodstuff; metabolic disorders; consumers; satisfaction. 


\section{Résumé}

Wavé-fortex est un complément alimentaire qui a été élaboré par l'université des Sciences et techniques de Masuku (USTM) au Gabon. Il a pour objectif de lutter contre les maladies du métabolisme, particulièrement, les impuissances, fatigues et faiblesses sexuelles, les hypertensions, la surcharge pondérale et les arythmies cardiaques. La présente étude été menée pour analyser les répartitions des consommations selon les types d'affections. Les résultats ont révélé que Wavé-fortex est majoritairement consommé, pour des problèmes de troubles sexuels $(41 \%)$, de fatigue générale $(20 \%)$ et d'hypertension artérielle (15\%). Suivant le genre, les hommes sont les principaux consommateurs à $73 \%$. Leur consommation est essentiellement due à des préoccupations de stabilité sexuelle $(51 \%)$, de fatigue générale (24\%) et d'hypertension (23\%). Chez les femmes, les préoccupations sont plutôt le surpoids (32\%) et 1'hypertension (29\%), avant les troubles sexuels $(21 \%)$. Wavé-fortex est un produit majoritairement consommé au Gabon (70\%), au Congo (14\%), au Cameroun (6\%) et en France $(2,5 \%)$, par des patients d'âge compris entre 30 et 70 ans.

Mots clés : Wavé-fortex ; alicament ; troubles métaboliques ; consommateurs; satisfaction.

\section{Introduction}

Wavé-fortex est un complément alimentaire (sélection de 36 plantes) conçu au Gabon, pour lutter contre des problèmes de malnutrition et de dérèglement du métabolisme humain (Lépengué et al. 2016). C'est un tonifiant efficace contre les impuissances sexuelles, les faiblesses et fatigues sexuelles ou généralisées du corps. Il contient des propriétés diurétique, purgative, antimicrobienne, anti oxydante, aphrodisiaque, antigrippale, régulatrice de la pression artérielle, réductrice de la surcharge pondérale, et stimulatrice de production de la testostérone et des spermatozoïdes (Lépengué et al. 2015).

Son projet de conception a été initié sur la base des études préliminaires prospectives réalisées tant au Gabon, que dans les neufs provinces du Gabon. Ces études ont montré que plus de $68 \%$ des personnes âgées de 45 ans souffraient d'au moins un des cinq troubles du métabolisme suivants : hypertension artérielle, impuissance faiblesse ou fatigue sexuelles, arythmies cardiaques, diabète et surcharge pondérale. Ces taux s'élevaient à 72\% pour des personnes âgées de plus de 53 ans (Lépengué et al. 2015).

Ces troubles constituent donc véritablement problème de santé publique, d'autant plus qu'ils affectent l'une des fonctions fondamentales de l'existence : la procréation. Ces pathologies demeurent par ailleurs difficiles à 
combattre, car contrairement aux maladies infectieuses (paludisme, tuberculose, filariose...) dont les agents pathogènes sont connus, les troubles du métabolisme découlent de l'évolution technologique :

- Alimentation quantitative et non diversifiée ;

- Sédentarisation et absence d'activités physiques ;

- Stress professionnels occasionnés par des obligations de productivité ;

- Insuffisances et perturbations des rythmes de sommeil.

Face à ces maladies, la médecine conventionnelle reste limitée, et les traitements proposés généralement inadaptés, car chroniques, et modifiant les comportements sociaux des patients. Par ailleurs tous ces traitements répétitifs, par effet de rémanence occasionnent à long terme des affections d'autres organes tels que le foie, ou les reins (Duraffourd et Lapraz, 2002).

C'est donc pour apporter une contribution phyto alimentaire à la résolution de cette problématique que le projet "Wavé-fortex " a été initié depuis 2008. Son objectif était de proposer une formulation biomédicale non agressive (minimisant les effets secondaires), élaborée exclusivement à partir des plantes alimentaires, et susceptibles d'être utilisée de façon continue sur de longues périodes. Wavé-fortex est aujourd'hui enregistré au titre d'invention scientifique nationale à l'Office Gabonais de la Propriété Intellectuelle (OGAPI), sous la référence GA20160002, et breveté par l'Organisation Africaine de la Propriété Intellectuelle (OAPI; code 121600267) (Lépengué et al., 2016).

Le présent travail présente quelques analyses préliminaires de consommation de ce complément alimentaire deux ans après son autorisation officielle de mise en vente sur le marché.

\section{Materiel Et Methodes}

\section{Materiel}

Le matériel utilisé est un échantillon de consommateurs composé de 69659 personnes consentantes, ayant acheté les flacons commerciaux de Wavé-fortex dans différents officines du Gabon, ou dans des universités et centres de recherche des autres pays africains. Cet échantillon ne concerne que les consommateurs coopératifs, qui ont donc volontairement accepté de participer à la présente enquête scientifique.

\section{Methodes}

L'analyse des paramètres de consommation de Wavé-fortex s'est basée sur l'exploitation des fiches commerciales de vente, qui mentionnent : le sexe du client, le pays d'achat (adresse) et le motif de consommation. Un site numérique et une page Facebook du produit permettent aux clients de donner leurs différents avis après consommation, afin d'évaluer les taux de satisfaction. 


\section{Analyses Statistiques}

Les analyses statistiques ont été réalisées avec un Logiciel Statistica 6.8. En cas de différence, les tests comparaisons multiples de Newman-keuls ont été utilisés au seuil de $5 \%$.

\section{Resultats}

\section{Repartition Des Consommation Par Pathologie}

L'analyse des ventes a révélé que 28456 flacons de Wavé-fortex (sur 69659) ont été achetés pour des besoins d'impuissances, de fatigues ou de faiblesses sexuelles. Ces ventes représentent $41 \%$ des stocks totaux du produit (Figure 1). Les demandes de Wavé-fortex sont dans un second temps liées aux problèmes de fatigues générales $(20 \%)$ d'hypertensions artérielles $(15 \%)$ et de surcharge pondérales $(10 \%)$. L'usage de Wavé-fortex pour des problèmes de ballonnements de ventre (7\%), ou de constipation (5\%) est relativement limité. Mais les usages les plus restreints de ce complément alimentaire sont ceux liés aux pathologies grippales, ou aux sinusites, avec des pourcentages de consommation moyens de $1 \%$. L'analyse statistique a montré qu'en dehors des deux dernières affections (sinusite et grippe), les différences des commandes entre pathologies se sont révélé significatives au seuil de 5\%.

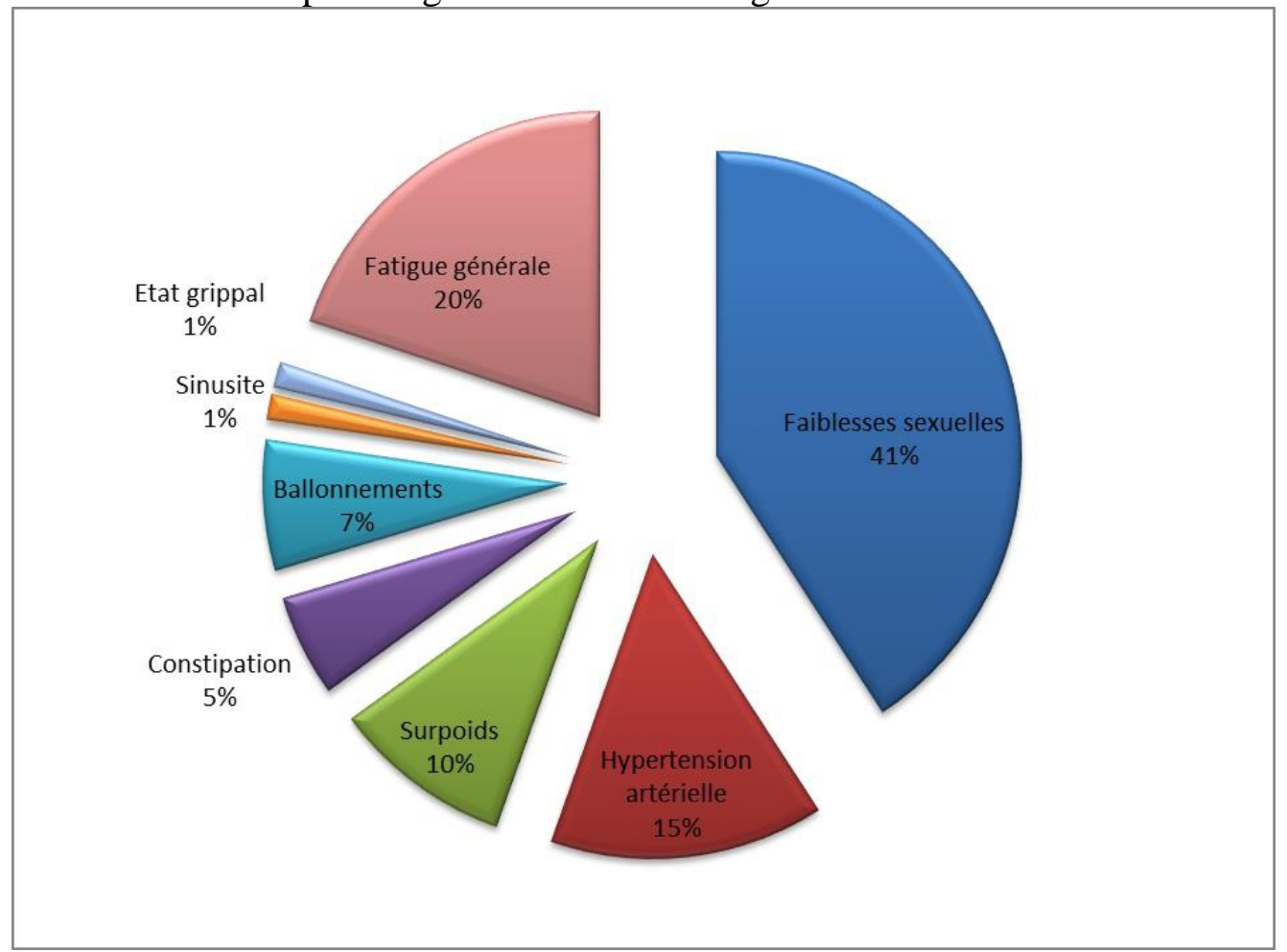

Figure 1 : Répartition des consommations de Wavé-fortex, suivant les maladies des usagers. 


\section{Analyse De La Satisfaction Des Consommateurs}

Les résultats de l'analyse des satisfactions des consommateurs sont présentés au tableau I. Leur analyse a révélé que les taux de satisfécit varient suivant les motifs d'usage du produit. Les consommateurs les moins satisfaits sont ceux qui ont utilisé le complément alimentaire, pour réduire les charges pondérales, avec un pourcentage de $68,44 \%$, soit 4567 consommateurs sur un total de 6673 personnes. Les taux de satisfaction sont en revanche très élevés chez les personnes ayant consommé Wavé-fortex pour des raisons d'hypertension artérielle (89,05\%), de faiblesses sexuelles $(92,16 \%)$, de constipation $(93,22 \%)$, et surtout de ballonnements de ventre $(95,52 \%)$. Ce complément alimentaire a également une bonne côte d'agrément chez les personnes en situation d'état grippal, avec un taux d'acceptation de 80,23\%.

Tableau I : Pourcentages de satisfaction des consommateurs de Wavé-fortex, selon les pathologies.

\begin{tabular}{|c|c|c|c|}
\hline Pathologie & Echantillon & Satisfaits & \% de satisfaction \\
\hline Faiblesses sexuelles & 28456 & 26225 & 92,1598257 \\
\hline Hypertension artérielle & 10142 & 9032 & 89,05541313 \\
\hline Surpoids & 6673 & 4567 & 68,43998202 \\
\hline Constipation & 3778 & 3522 & 93,223928 \\
\hline Ballonnements & 4804 & 4589 & 95,52456286 \\
\hline Sinusite & 956 & 767 & 80,23012552 \\
\hline Etat grippal & 974 & 882 & 90,55441478 \\
\hline Fatigue générale & 13876 & 12446 & 89,69443644 \\
\hline
\end{tabular}

\section{Repartiton Des Consommations Suivant Le Genre}

Wavé-fortex est un complément alimentaire, qui a majoritairement été consommé par le genre masculin, avec 50956 flacons achetés (sur 69659 unités), équivalent à un pourcentage de $73 \%$. Les femmes avec un total de 18703 flacons achetés n'ont représenté donc que $27 \%$ de l'échantillonnage des consommateurs (Figure 2). Cette différence de consommation est significative au seuil de $5 \%$.

Chez les hommes, la consommation de Wavé-fortex a essentiellement été due aux préoccupations des faiblesses, fatigues ou impuissances sexuelles (51\%), de fatigues générales (24\%), et d'hypertension (23\%) (Figure 3). Chez les femmes, en revanche, l'achat du produit reposait d'abord sur les préoccupations de réduction des charges pondérales (32\%) et d'hypertension 
artérielle (29\%), avant celles des fatigues ou faiblesses sexuelles (21\%) (Figure 4). Chez les deux genres, l'utilisation de Wavé-fortex pour les pathologies grippales et la sinusite a été faible et inférieure à $2 \%$ des ventes.

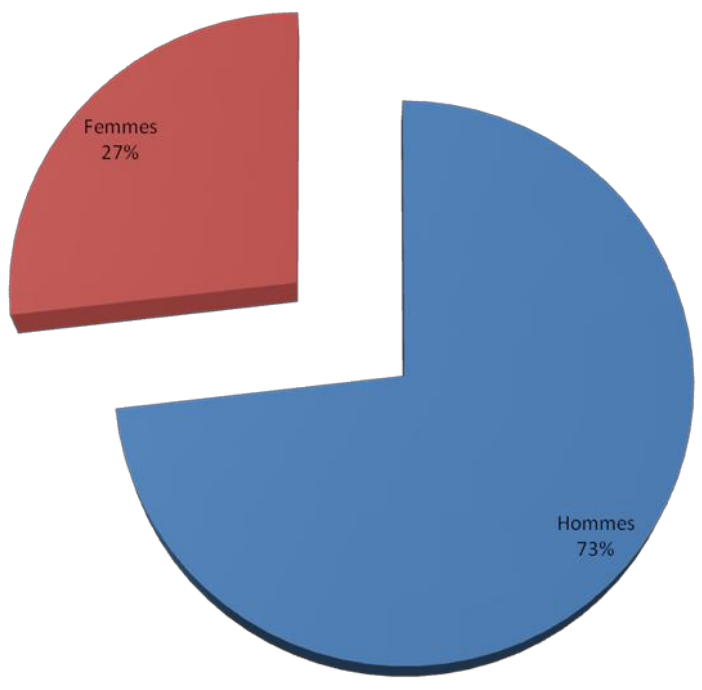

Figure 2 : Répartition des consommations de Wavé-fortex, suivant les genres.

\section{HOMMES}
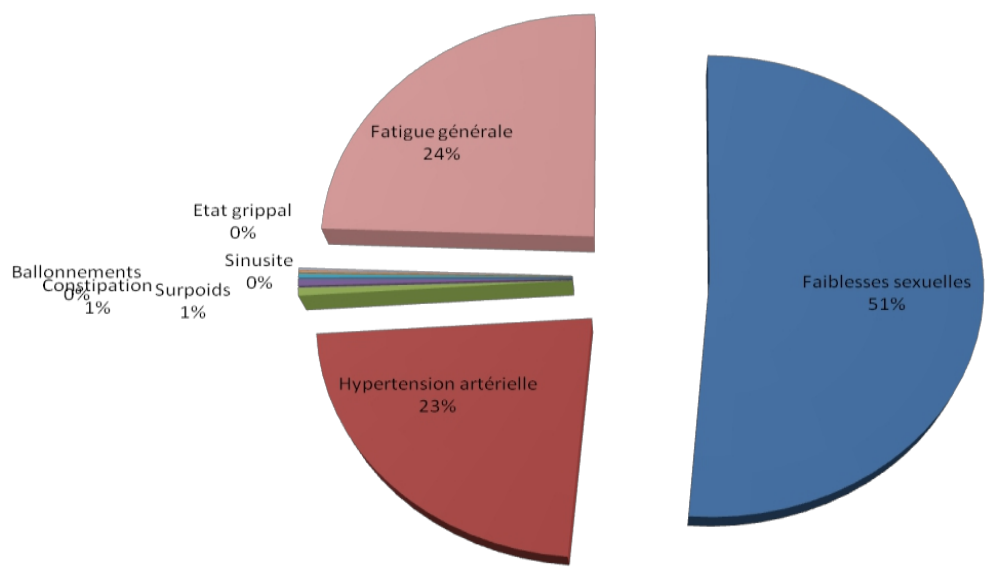
A

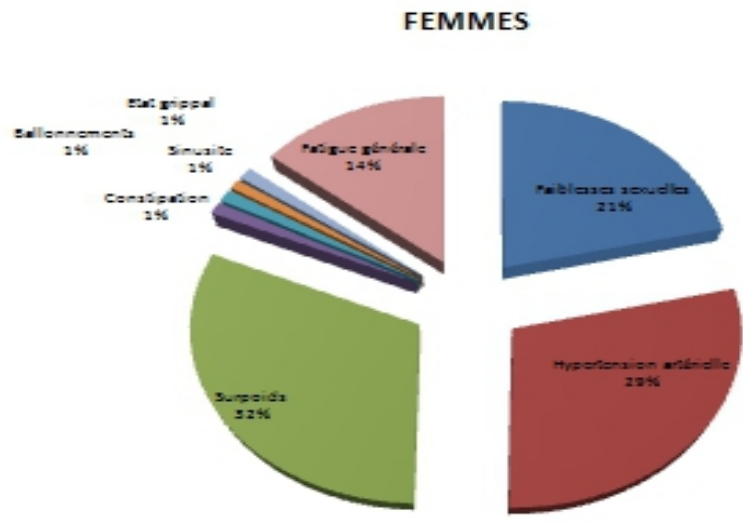

B

Figure 3 : Répartition des consommations de Wavé-fortex suivant les maladies déclarées chez les hommes (A) et chez les femmes (B).

\section{Analyse Des Ages Des Consommateurs}

Suivant les âges des consommateurs, les résultats obtenus (figure 4) ont révélé que les plus fortes demandes provenaient des patients deux sexes âgés de 50 à 60 ans ( $34 \%$ d'achat, soit 23653 flacons vendus). L'analyse de ces résultats a également montré que les tranches d'âges respectifs de 40 à 50 ans et de 30 à 40 ans étaient également très concernées, avec des taux de consommation respectifs de 26\% (18324 flacons) et 17\% (11752 flacons). Les achats de Wavé-fortex ont en revanche été faibles pour les patients d'âge inférieur à 20 ans $(5 \%)$ ou supérieur à 70 ans $(3 \%)$. L'analyse statistique a montré l'existence de différences significatives de consommation du produit entre les différences tranches d'âge. 


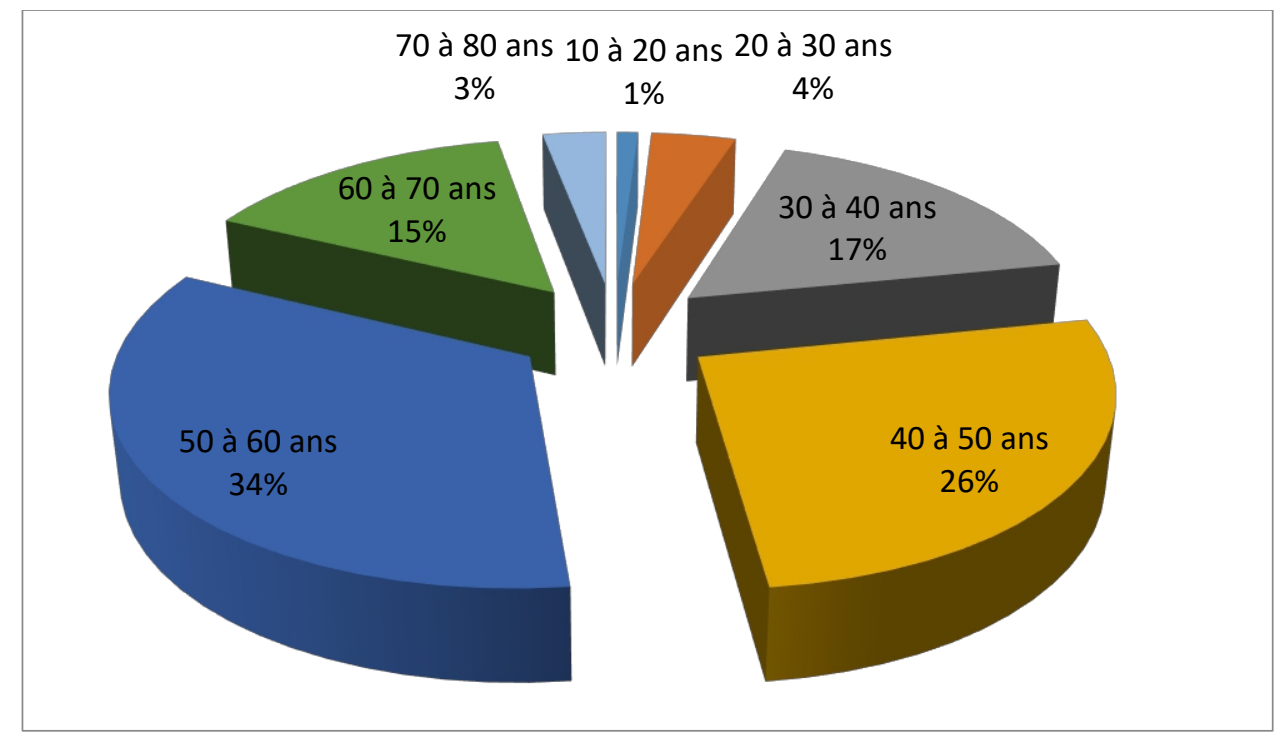

Figure 4 : Répartition des consommations de Wavé-fortex suivant les tranches d'âge des demandeurs.

\section{Analyse Des Consommations Par Pays}

Avec 48238 acheteurs (sur 69659), le Gabon a été le principal pays de consommation de Wavé-fortex, (soit un pourcentage de 69,25\%) (Figure 5). Cette consommation est significativement supérieure à celle de tous les autres pays rassemblés. Le Congo et le Cameroun se sont classé respectivement second et troisième pays consommateurs, avec 9867 et 4476 flacons achetés. Ces quantités correspondent à des taux de vente respectifs de $14,16 \%$ et $6,43 \%$. La consommation de Wavé-fortex dans d'autres pays a été jugée faible, notamment en France et en Guinée Equatoriale (2\%), mais surtout au Burkina Faso, en Côte d'Ivoire, au Sénégal et en République Démocratique du Congo, avec des pourcentages d'achat moyens de $1 \%$.

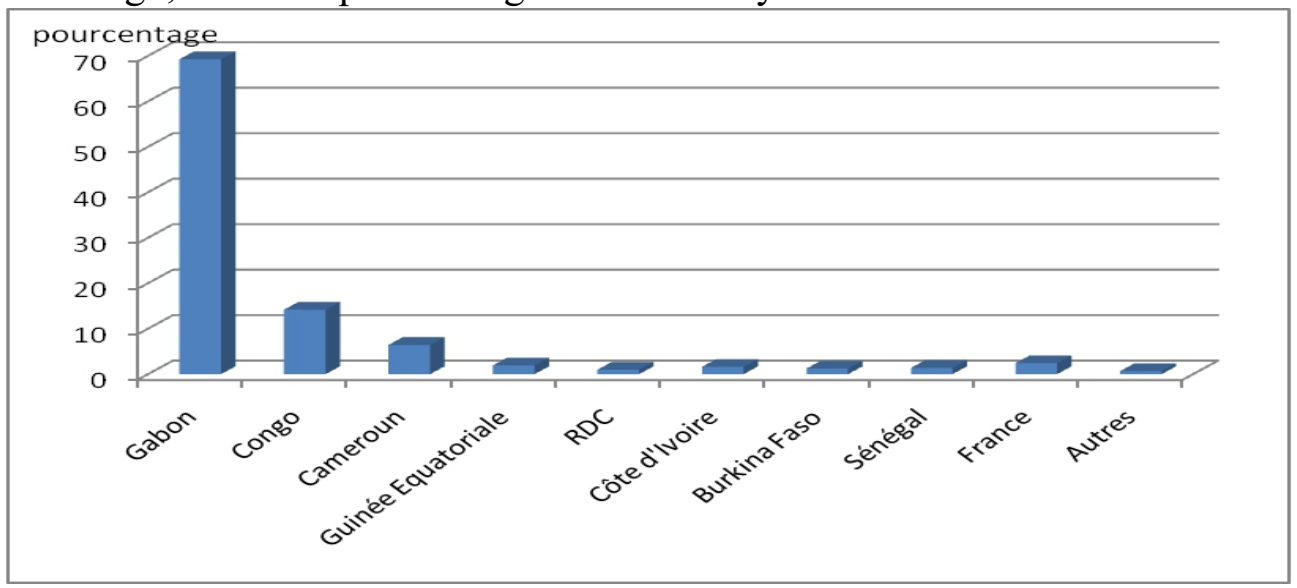

Figure 5 : Répartition des consommations de Wavé-fortex, suivant les nationalités des usagers. 


\section{Discussion}

Les résultats de cette étude ont montré que sur un échantillon total 69659 consommateurs de Wavé-fortex ayant participé à l'enquête, la quête de résolution des problèmes d'impuissance, faiblesse ou la fatigue sexuelle reste la préoccupation majeure des populations. En effet, $41 \%$ des consommations sont liés à cette préoccupation. Ces taux sont particulièrement élevés chez les hommes, lorsqu'on considère la répartition des besoins par genre (51\%). Les présents résultats sont en accord avec les travaux de Lépengué et al. (2015) qui ont rapporté des troubles précoces de métabolisme (hypertension artérielle, impuissance fatigue ou faiblesse sexuelle, arythmies cardiaques, diabète, surcharge pondérale...) chez les hommes et femmes d'âge supérieur à 45 ans. Ces troubles paraissent occasionnés par diverses sources, dont les modifications alimentaires favorisant les consommations grasses, les pressions et stress professionnels, les pollutions environnementales etc. (Lépengué et al. 2017).

Parmi les 28456 personnes, ayant consommé Wavé-fortex, pour des troubles de sexualité, 26225 sujets ont déclaré avoir été satisfaits; ce qui correspond à un taux de satisfaction de 92,16\%. Ces résultats sont parfaitement en accord avec ceux mentionnés par Lépengué et al. (2015). Le mode d'action de Wavé-fortex repose sur la spécificité de ses composants nutritionnels. En effet, ce produit est riche en éléments minéraux, notamment le zinc et le calcium, ainsi qu'en diverses vitamines (A, B $1, B_{3}, B_{6}, C$ et $\left.\mathrm{K}\right)$. Le zinc est connu pour être un stabilisateur de la testostérone, par son action inhibitrice sur l'aromatase, l'enzyme de dégradation de cette hormone sexuelle (Bruneton, 2009). Les vitamines B6 sont également impliquées dans la production de cette hormone (Bradley, 2006). Le calcium et l'acide folique sont aussi connus pour leur intervention dans les processus d'amélioration de la spermatogénèse (Huguet, 1999). L'action combinée de tous ces composés pourrait être à l'origine de la revitalisation sexuelle rapportée par les consommateurs de ce complément alimentaire.

Wavé-fortex a également montré une bonne efficacité contre les fatigues généralisées, avec des taux de satisfaction de 89,70\%. Ces résultats sont sans doute liés à la richesse minérale du produit, notamment en calcium (12,6 mg/g de MS), en zinc ( $0,12 \mathrm{mg} / \mathrm{g}$ de MS), en fer $(0,94 \mathrm{mg} / \mathrm{g}$ de MS), et en potassium ( $8,2 \mathrm{mg} / \mathrm{g}$ de MS) (Lépengué et al., 2016). Avec une charge calorifique de $355,88 \mathrm{Kcal}$ pour $100 \mathrm{~g}$ de MS, Wavé-fortex est un produit très énergétique, malgré sa faiblesse en composés lipidiques (Lépengué et al. 2017). Wavé-fortex contient également plusieurs vitamines, dont la vitamine A (2,3 mg/g de MS), la vitamine B3 (3,6 mg/g de MS), la vitamine B6 (2,1 $\mathrm{mg} / \mathrm{g}$ de MS), la vitamine C (193 mg/g de MS) et la vitamine $\mathrm{E}(43,2 \mathrm{mg} / \mathrm{g}$ de MS) (Lépengué et al., 2016). 
Les résultats de cette étude ont aussi révélé des forts taux de satisfaction contre l'hypertension artérielle. En effet, sur 10142 consommateurs, 9032 personnes se sont déclaré satisfaites du traitement ; ce qui donne à un taux d'agrément proche de $90 \%$. Ces résultats concordent avec ceux rapportés par Lepengué et al. (2015). La réduction de la pression artérielle chez les sujets malades pourrait provenir des composés vasodilatateurs, dont les vitamines B3, qui augmentent le diamètre des vaisseaux sanguins, par relâchement des muscles lisses (Wichtl et Anton, 2003). Cette action permet alors une meilleure fluidité de la circulation sanguine, abaissant ainsi la pression du sang, donc l'hypertension artérielle. La richesse de ce complément alimentaire en potassium $(\mathrm{K}+)$ pourrait aussi expliquer l'action efficace observée contre l'hypertension artérielle. En effet, les ions potassium $\mathrm{K}^{+}$, par le fonctionnement des pompes ATPases électrogènes situées sur les membranes cellulaires, abaissent la pression artérielle des vaisseaux, par son échange en déséquilibre de charges ave le sodium $\left(\mathrm{Na}^{+}\right)$(Ursell, 2001).

Les disproportions de consommation entre divers pays pourraient s'expliquer par la situation géographique des unités de productions qui sont toutes installées au Gabon. Les consommations du Congo (14\%) et du Cameroun $(6,43 \%)$ semblent s'expliquer par la proximité géographique et culturelle de ces deux limitrophes du Gabon. La consommation surprenante de la France $(2,4 \%)$ provient essentiellement des diasporas africaines, et surtout des délégations sportives ayant couvert les festivités de la Coupe d'Afrique des Nations (CAN) organisée au Gabon, entre les mois de janvier et février 2017

\section{Conclusion}

Wavé-fortex est un complément alimentaire efficace contre plusieurs dérèglements du métabolisme humain. Il est essentiellement consommé pour des problèmes de fatigue, faiblesse et impuissances sexuelles. Il est aussi dans une large mesure acheté pour des préoccupations de fatigues généralisées et d'hypertension artérielle. Sa consommation reste majoritairement masculine et gabonaise. Les analyses d'enquêtes effectuées chez les consommateurs révèlent globalement des taux de satisfaction supérieurs à $90 \%$. Ce qui est fortement intéressant pour l'organisme humain, en raison de la constitution totalement alimentaire de ce produit.

\section{References:}

1. Bradley P. British Herbal Compendium : A handbook of scientific information on widely used plant drugs ; Companion to the British herbal Pharmacopoeia. British Herbal Medicine Association (BHMA), Bournemouth (UK), 487 p, 2006. 
2. Bruneton J. Pharmacognosie-Phytochimie: Plantes médicinales. 3ème édition Tech \& Doc - médicales internationales, Paris, 1120 p, 2002.

3. Bruneton J. Pharmacognosie - Phytochimie, plantes médicinales. 4e édition Tech \& Doc - médicales internationales, Paris, 1288 p, 2009.

4. Duraffourd C, Lapraz JC. Traité de phytothérapie clinique, Médecine et Endobiogénie. Edition Masson, Paris (FR), 204 p, 2002.

5. Fleurentin J. Les plantes qui nous soignent: traditions et thérapeutiques. Editions Ouest-France, Rennes 380 p, 2007.

6. Girre L. Traditions et propriétés des plantes médicinales : Histoire de la pharmacopée. Editions de santé Privat, Toulouse, 378 p, 1997.

7. Huguet C. Le silicium In: Les oligo-éléments en médecine et biologie. Edition Tech \& Doc 1: 609-624, 1999.

8. Lepengué AN, Mavoungou JF, Souza A, M'batchi B. Propriétés de Wavé fortex, un médicament efficace, à base des plantes alimentaires du Gabon. $2^{\mathrm{e}}$ Journées scientifiques du CAMES (Exposition-vente); Dakar du 19 au 21 novembre 2015.

9. Lepengué AN, Souza A, Yala JF, Lebamba J, Mavoungou JF, M'batchi B. Etude de quelques caractéristiques physicochimiques et biochimiques de Wavé-fortex, un complément alimentaire naturel du Gabon. European Scientific Journal 12(33) : 508-520

10. Lepengué AN. Etude de Wavé-fortex, un complément alimentaire du Gabon. Actes des $3^{\mathrm{e}}$ Journées Scientifiques du CAMES; PTR Sécurité Alimentaire, 1146 p, 2017.

11. Ursell A. Guide pratique des vitamines et minéraux. Editions Hachette, Montréal, Québec, 128 p, 2001.

12. Wichtl M, Anton R. Plantes thérapeutiques: Traditions, pratique officinale, science et thérapeutique. $2^{\mathrm{e}}$ Éditions Tech \& Docmédicales internationales, Paris 208 p, 2003. 